\title{
Mobilization of the nonconjugative virulence plasmid from hypervirulent Klebsiella pneumoniae
}

Yanping $\mathrm{Xu}^{1+}$, Jianfeng Zhang ${ }^{2+}$, Meng Wang ${ }^{2}$, Meng Liu², Guitian Liu ${ }^{2}$, Hongping $\mathrm{Qu}^{3}$, Jialin Liư ${ }^{3}$, Zixin Deng ${ }^{2}$, Jingyong Sun ${ }^{4,5^{*}}$, Hong-Yu Ou ${ }^{2^{*}}$ and Jieming $\mathrm{Qu}^{1^{*}}$ (D)

\begin{abstract}
Background: Klebsiella pneumoniae, as a global priority pathogen, is well known for its capability of acquiring mobile genetic elements that carry resistance and/or virulence genes. Its virulence plasmid, previously deemed nonconjugative and restricted within hypervirulent K. pneumoniae (hvKP), has disseminated into classic K. pneumoniae (CKP), particularly carbapenem-resistant K. pneumoniae (CRKP), which poses alarming challenges to public health. However, the mechanism underlying its transfer from hvKP to CRKP is unclear.

Methods: A total of 28 sequence type (ST) 11 bloodstream infection-causing CRKP strains were collected from Ruijin Hospital in Shanghai, China, and used as recipients in conjugation assays. Transconjugants obtained from conjugation assays were confirmed by Xbal and S1 nuclease pulsed-field gel electrophoresis, PCR detection and/or whole-genome sequencing. The plasmid stability of the transconjugants was evaluated by serial culture. Genetically modified strains and constructed mimic virulence plasmids were employed to investigate the mechanisms underlying mobilization. The level of extracellular polysaccharides was measured by mucoviscosity assays and uronic acid quantification. An in silico analysis of 2608 plasmids derived from 814 completely sequenced $K$. pneumoniae strains available in GenBank was performed to investigate the distribution of putative helper plasmids and mobilizable virulence plasmids.
\end{abstract}

\footnotetext{
*Correspondence: 13671578899@126.com; hyou@sjtu.edu.cn; jmqu0906@163.com

'Yanping $\mathrm{Xu}$ and Jianfeng Zhang shared the first authorship.

${ }^{4}$ Department of Laboratory Medicine, Ruijin Hospital, Shanghai Jiao Tong University School of Medicine, Shanghai 200025, China

${ }^{2}$ State Key Laboratory of Microbial Metabolism, Joint International Laboratory on Metabolic \& Developmental Sciences, School of Life Sciences \& Biotechnology, Shanghai Jiao Tong University, Shanghai 200030, China ${ }^{1}$ Department of Pulmonary and Critical Care Medicine, Ruijin Hospital, Shanghai Jiao Tong University School of Medicine, Shanghai 200025, China; Institute of Respiratory Diseases, Shanghai Jiao Tong University School of Medicine, Shanghai 200025, China; Shanghai Key Laboratory of Emergency Prevention, Diagnosis and Treatment of Respiratory Infectious Diseases, Shanghai 200025, China

Full list of author information is available at the end of the article
}

C C The Author(s). 2021 Open Access This article is licensed under a Creative Commons Attribution 4.0 International License, which permits use, sharing, adaptation, distribution and reproduction in any medium or format, as long as you give appropriate credit to the original author(s) and the source, provide a link to the Creative Commons licence, and indicate if changes were made. The images or other third party material in this article are included in the article's Creative Commons licence, unless indicated otherwise in a credit line to the material. If material is not included in the article's Creative Commons licence and your intended use is not permitted by statutory regulation or exceeds the permitted use, you will need to obtain permission directly from the copyright holder. To view a copy of this licence, visit http://creativecommons.org/licenses/by/4.0/ The Creative Commons Public Domain Dedication waiver (http://creativecommons.org/publicdomain/zero/1.0/) applies to the data made available in this article, unless otherwise stated in a credit line to the data. 
Results: A nonconjugative virulence plasmid was mobilized by the conjugative plasmid belonging to incompatibility group F (InCF) from the hvKP strain into ST11 CRKP strains under low extracellular polysaccharideproducing conditions or by employing intermediate E. coli strains. The virulence plasmid was mobilized via four modes: transfer alone, cotransfer with the conjugative IncF plasmid, hybrid plasmid formation due to two rounds of single-strand exchanges at specific 28-bp fusion sites or homologous recombination. According to the in silico analysis, 31.8\% (242) of the putative helper plasmids and 98.8\% (84/85) of the virulence plasmids carry the 28-bp fusion site. All virulence plasmids carry the origin of the transfer site.

Conclusions: The nonconjugative virulence plasmid in ST11 CRKP strains is putatively mobilized from hvKP or E. coli intermediates with the help of conjugative IncF plasmids. Our findings emphasize the importance of raising public awareness of the rapid dissemination of virulence plasmids and the consistent emergence of hypervirulent carbapenem-resistant K. pneumoniae (hv-CRKP) strains.

Keywords: Klebsiella pneumoniae, Virulence plasmid, IncF plasmid, Conjugation, Mobilization

\section{Background}

Klebsiella pneumoniae, a common cause of hospital- and community-acquired infections, has increasingly gained public attention due to its capability of acquiring new plasmids and other mobile genetic elements that carry resistance- and/or virulence-associated genes [1-3]. $K$. pneumoniae strains can generally be classified into classical K. pneumoniae (cKP) and hypervirulent K. pneumoniae (hvKP) [4]. These two pathotypes can be distinguished by their disease profiles and genetic characteristics. cKP commonly causes infections in healthcare settings and carries plasmid(s) coding for antimicrobial resistance, and sequence type (ST) 11 carbapenem-resistant K. pneumoniae (CRKP) is the most prevalent $K$. pneumoniae strain in China. hvKP is typically associated with invasive diseases within the community and frequently harbours a virulence plasmid, which is the major pathogenic determinant of hypermucoviscosity and hypervirulence phenotypes [4], such as the well-documented virulence plasmid pLVPK of $K$. pneumoniae CG43 (GenBank accession number AY378100) [5] that encodes the mucoid regulators RmpA, aerobactin and salmochelin [6]. Potential biomarkers (including peg-344, iroB, іисA, rmpA and rmpA2 as well as increased siderophore production in hvKP) were previously identified for the accurate differentiation of these two pathotypes [7]. Recently, K. pneumoniae isolates with genes conferring hypervirulence and multidrug resistance and even carbapenem resistance have increasingly emerged, which have caused bacteremia, metastatic infection and even death $[8,9]$. These variants potentially developed in two directions [4, 9]: carbapenemresistant hvKP (CR-hvKP), an hvKP acquiring a plasmid encoding carbapenemase, and hypervirulent carbapenem-resistant $K$. pneumoniae (hv-CRKP), a CRKP strain acquiring a virulence plasmid. Studies focusing on the transmission of self-transferable carbapenem-resistant plasmids have provided powerful support for the possibility that hvKP evolved into CRhvKP $[2,10]$. However, the virulence plasmids of $K$. pneumoniae are generally regarded as nonconjugative, and few studies have investigated the transfer of $K$. pneumoniae virulence plasmids.

Several recent studies have discovered resistance and virulence hybrid plasmids in $K$. pneumoniae of various sequence types, including hvKP clone types (e.g. ST23) [11] and cKP clone types (e.g. ST11, ST15, ST101 and ST147) $[8,12-14]$. Some of these hybrid plasmids are combinations of conjugative resistance plasmids belonging to incompatibility group $\mathrm{F}$ ( $\mathrm{IncF}$ ) and virulence plasmids [2]. Yang et al. discovered such a hybrid plasmid in a clinical Klebsiella variicola strain and confirmed its self-transferable ability [15]. Another study observed homologous recombination between a virulence plasmid and an IncF1A plasmid in a clinical $K$. pneumoniae strain, which yielded a conjugative hybrid plasmid [16]. Li et al. reported that a non-pLVPK-like virulence plasmid could be transferred alone [17], but its helper plasmid and the corresponding mechanism remain to be uncovered. Notably, these studies focused on the existing conjugative hybrid plasmids in cKP or $K$ variicola but did not reveal the mechanism underlying the transmission of pLVPK-like nonconjugative virulence plasmids from hvKP to cKP, particularly ST11 CRKP [2].

We previously reported the hypervirulent $K$. pneumoniae strain RJF293 of capsular serotype K2 (accession number PRJNA307277) [18], which caused clinical metastatic infection, and confirmed its hypervirulence phenotype using a mouse lethality assay [19]. This strain carries the 224,263-bp virulence plasmid pRJF293 with high nucleotide sequence similarity ( $95 \%$ query coverage and $99 \%$ identities) to the classic virulence plasmid pLVPK. Here, we employed this pLVPK-like nonconjugative virulence plasmid and its variants to investigate the mechanism underlying virulence plasmid transfer. We found that the virulence plasmid could be 
transferred from hvKP to ST11 CRKP and E. coli strains with the help of a self-transferable IncF plasmid. We also identified four modes of virulence plasmid mobilization, including transfer with or without the conjugative IncF plasmid and fusion with the IncF plasmid via homologous recombination or two rounds of single-strand exchanges at specific 28-bp fusion sites. In this study, we also confirmed that virulence plasmid transfer from hvKP to CRKP could be limited by the overproduction of extracellular polysaccharides, which can be achieved by reducing extracellular polysaccharide production or employing $E$. coli strains as the intermediate vector. The in silico analysis revealed the wide distribution of putative conjugative helper plasmids and mobilizable virulence plasmids, which indicates that virulence plasmids might rapidly disseminate along with IncF plasmids that frequently carry carbapenemase genes.

\section{Methods}

\section{Bacterial strains}

For the conjugation assays (see below), the previously reported hvKP RJF293 [18] and its derivatives were employed as donor strains. Twenty-eight bloodstream infection-causing ST11 CRKP strains as well as ST11 CRKP HS11286 [20], its derivative HS11286pKPHS2 $\triangle$ oriT, and E. coli 553 and C600 were employed as recipient strains. All 28 ST11 CRKP strains were isolated from blood culture samples collected for routine clinical examinations of hospitalized patients admitted to Ruijin Hospital in Shanghai, China, from 2018 to 2019. The strains and plasmids used in this study are listed in Additional file 1: Table S1.

\section{Construction of genetically modified strains}

For gene mutation or insertion, as described previously $[10,20]$, we replaced the target gene with the hygromycin B phosphotransferase gene $(h p h)$ flanked by flippase recognition sites (FRT) or inserted the $h p h$ gene via lambda red recombination. The $h p h$ gene conferring hygromycin resistance can be subsequently removed by Flp-FRT recombination. Details are provided in Additional file 2: Supplementary Methods. The primers used in this study are listed in Additional file 3: Table S2.

\section{Conjugation assay}

Following overnight culture, both the donor and recipient strains were cultured at $220 \mathrm{rpm}$ and $37^{\circ} \mathrm{C}$ to the logarithmic phase of growth $\left(\mathrm{OD}_{600}\right.$ approximately 0.6$)$ in lysogeny broth (LB) media. One millilitre of donor cells and recipient cells was washed with $\mathrm{PBS}$, resuspended in $20 \mu \mathrm{l}$ of $10 \mathrm{mM} \mathrm{MgSO}_{4}$, mixed and then inoculated on LB agar plates. After overnight culture at $37^{\circ} \mathrm{C}$, the bacteria were resuspended and serially diluted in PBS and spread on antibiotic-containing LB agar plates for transconjugant selection. The antibiotic and the corresponding concentration used for each pair of conjugates are listed in Additional file 4: Table S3. The transconjugants were further validated by $\mathrm{XbaI}$ and $\mathrm{S} 1$ nuclease pulsed-field gel electrophoresis (PFGE) combined with PCR detection. According to the colonyforming unit (CFU) count on the serial dilution plates containing corresponding antibiotics, the conjugation frequency was calculated as the ratio of transconjugants to recipients.

\section{Whole-genome sequencing (WGS) and annotation}

The genomic DNA of RJBSI76, RJBSI76-pV, J53-p1-pVhybrid-1 and XL10-pF-pV-hybrid-1 was extracted and then sequenced using the combination of the 150-bp paired-end Illumina NovaSeq 6000 platform and the PacBio RSII single-molecule long-read sequencing platform. The trimmed and filtered reads were de novo assembled using Canu 2.0 [21]. The genome sequences are deposited in the National Center for Biotechnology Information (NCBI) BioProject repository under the accession numbers PRJNA681750 [22], PRJNA682095 [23], PRJNA692573 [24] and PRJNA692574 [25]. The genomic data were annotated with Prokka 1.1.3 [26]. PlasmidFinder 2.1 [27] was used to determine the plasmid incompatibility types, and Kleborate [28] was used to determine the sequence type of the strains. The putative virulence factors, antibiotic resistance determinants, insertion sequences (ISs) and other mobile genetic elements were predicted using VRprofile [29]. The conjugative transfer-related modules of the plasmids, including the relaxase gene, the type IV coupling protein (T4CP) gene and the tra gene cluster for the type IV secretion system (T4SS), were detected by oriTfinder [30]. Alignments of the plasmid sequences were performed using BLAST Ring Image Generator (BRIG) [31] and Easyfig [32].

\section{Construction of the mimic virulence plasmid}

First, the origin of transfer (oriT) region of the virulence plasmid pRJF293HA was detected using the BLASTn searches against the back-end database of oriTfinder, oriTDB [30], with a high BLAST E-value of 1.0. The subsequent manual curation of the conserved nick site (nic) and the flanking putative inverted repeats (IRs) located within the oriT region was performed using MEME-MAST [33] and Vmatch (http://vmatch.de/), respectively. The predicted oriT of pRJF293HA was amplified, digested with BamHI and HindIII and then inserted into the plasmid pACYC184-Apr. The constructed plasmid pACYC184-Apr-ori $T_{\mathrm{RJF} 293 \mathrm{HA}}$ was introduced into the E. coli strains XL10-pF and C600-p1 as well as their genetically modified strains with calcium chloride treatment. The transfer of the mimic virulence plasmid 
pACYC184-Apr-ori $T_{\mathrm{RJF} 293 \mathrm{HA}}$ was validated by Sanger sequencing of the PCR product and PFGE using XbaI restriction.

\section{Plasmid stability in transconjugants}

A single colony of each transconjugant was picked up from the freshly streaked agar plate and inoculated into fresh LB broth. Serial culture of each purified transconjugant was performed for 2 weeks. Ten microlitres of the bacterial suspension was transferred to $10 \mathrm{ml}$ of fresh LB broth every $12 \mathrm{~h}$. The plasmid stability was assessed by streaking each subculture of transconjugants on fresh LB agar plates and randomly selecting three single colonies for antibiotic resistance verification and PCR detection of the iro $B$ gene of the virulence plasmid and the traE gene of the IncF plasmid. S1 nucleasePFGE was performed with one of the three validated colonies.

\section{Mucoviscosity assay}

As previously described, the mucoviscosity was determined using a sedimentation assay with some modifications [34]. Overnight cultures of $K$. pneumoniae strains grown in LB broth were subcultured to an $\mathrm{OD}_{600}$ of 0.2 in fresh $\mathrm{LB}$ broth and grown at $37^{\circ} \mathrm{C}$ for $4 \mathrm{~h}$. The bacterial cultures were normalized to an $\mathrm{OD}_{600}$ of $1.0 / \mathrm{ml}$ and aspirated to $2 \mathrm{ml}$ for sedimentation at $1000 \times g$ or $2500 \times$ $g$ for $5 \mathrm{~min}$. The top $200 \mu \mathrm{l}$ of the supernatant was carefully removed without disturbing the pellet for $\mathrm{OD}_{600}$ measurement.

\section{Capsule extraction and quantification assay}

The extraction and quantification of uronic acid were performed as previously described with some modifications [34]. Overnight cultures grown in LB broth were subcultured to an $\mathrm{OD}_{600}$ of 0.2 in fresh $\mathrm{LB}$ medium and grown at $37^{\circ} \mathrm{C}$ for $4 \mathrm{~h}$. Five hundred microlitres of bacterial culture was mixed with $100 \mu \mathrm{l}$ of $1 \%$ Zwittergent$100 \mathrm{mM}$ citric acid, and the mixture was incubated for $20 \mathrm{~min}$ at $50^{\circ} \mathrm{C}$. The cells were pelleted by centrifugation, and $300 \mu \mathrm{l}$ of the supernatant was aspirated into $1.2 \mathrm{ml}$ of absolute ethanol, incubated for $20 \mathrm{~min}$ at $4^{\circ} \mathrm{C}$ and centrifuged at maximum speed for $5 \mathrm{~min}$. The pellet was dried, resuspended in $200 \mu \mathrm{l}$ of distilled water and mixed with $1.2 \mathrm{ml}$ of $12.5 \mathrm{mM}$ sodium tetraborate in sulfuric acid, and the mixture was incubated for $5 \mathrm{~min}$ at $100^{\circ} \mathrm{C}$ and then cooled on ice for $10 \mathrm{~min}$. The absorbance at $520 \mathrm{~nm}$ was measured after the addition of $20 \mu \mathrm{l}$ of $0.15 \% 3$-phenylphenol in $0.5 \% \mathrm{NaOH}$. The glucuronic acid content was determined according to the standard curve for glucuronic acid and expressed as micrograms per OD unit.

\section{Bioinformatic analysis of sequenced plasmids of $K$. pneumoniae}

As of January 28, 2021, 814 completely sequenced $K$. pneumoniae strains, including 2608 plasmids, were available in GenBank, and the accession numbers and genetic characteristics of these $2608 \mathrm{~K}$. pneumoniae plasmids are listed in Additional file 5: Table S4. Plasmids that contain the oriTfinder-predicted oriT region and genes encoding relaxase, T4CP and T4SS were defined as putative conjugative plasmids and were included in the subsequent analysis. The sequenced plasmids containing the full-length rmpA (or rmpA2) gene and the iuc gene cluster were defined as putative virulence plasmids because the presence of these genes was sufficient for the host strain to exhibit some extent of hypervirulence, as previously reported [4]. The details of the selected putative conjugative plasmids and putative virulence plasmids are provided in Additional file 6: Table S5 and Additional file 7: Table S6, respectively. The 28-bp fusion site was identified using the matchPattern function in the Biostrings $\mathrm{R}$ package with the specific sequence 'AGATCCGNAANNNNNNNNTTNCGGATCT'. The oriT region and genes encoding relaxase, T4CP and T4SS were predicted using oriTfinder [30]. The sequence type of the strains, antimicrobial resistance gene and virulence gene were determined using Kleborate [28]. PlasmidFinder 2.1 [27] was used to determine the plasmid replicon types. The phylogenetic analysis was constructed using OrthoMCL [35], and the tree was drawn using iTOL [36].

\section{Statistical analysis}

The data are presented as the means \pm standard deviations (SDs) based on three independent experiments. The difference in average values between the two groups was assessed by unpaired two-sided Student's $t$-test. A $P$ value less than 0.05 was considered to indicate significance. The data analyses were performed using the $R$ package (https://www.r-project.org/).

\section{Results}

Transfer of the nonconjugative virulence plasmid from hvKP to CRKP and further to E. coli

To clarify the mechanism underlying virulence plasmid mobilization, RJF293C containing the pLVPK-like nonconjugative virulence plasmid pRJF293C was employed as the donor. Twenty-eight bloodstream infectioncausing ST11 CRKP strains as well as ST11 CRKP HS11286 [20], its derivative HS11286-pKPHS2 $\Delta$ oriT, and E. coli $\mathrm{J} 53$ and C600 were employed as the individual recipients for conjugation. However, the transmission of pRJF293C was not observed in each conjugation pair. Because the rmpA gene contributes to the overproduction of extracellular polysaccharides, which might 


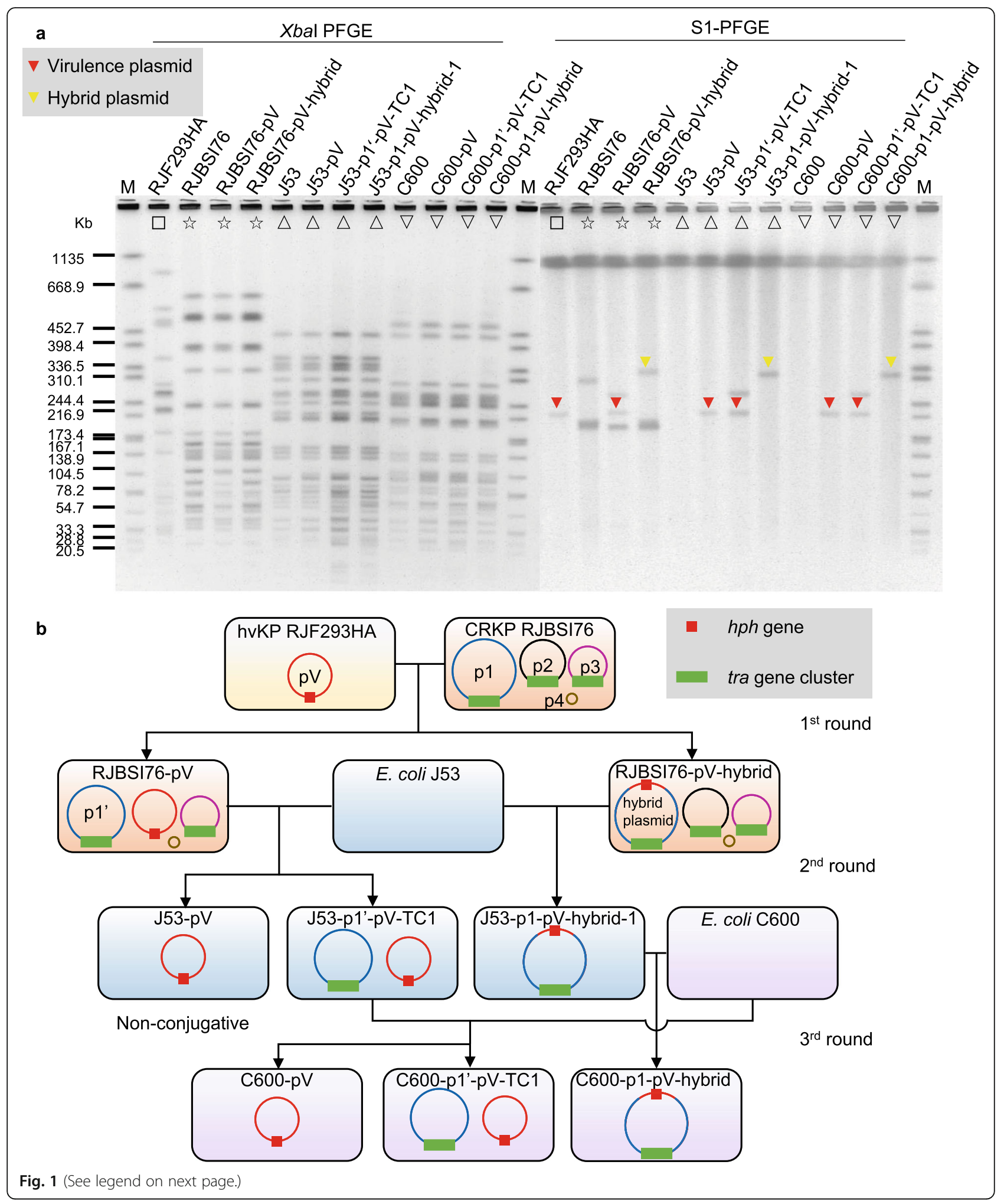


(See figure on previous page.)

Fig. 1 Transfer of the nonconjugative virulence plasmid from hvKP to carbapenem-resistant K. pneumoniae and E. coli. a Xbal PFGE and S1-PFGE of K. pneumoniae and E. coli transconjugants and their parental strains. Full details of the strains are provided in Additional file 1: Table S1. M represents the molecular weight marker Salmonella serotype Braenderup H9812 strain. Red triangles denote the virulence plasmid pRJF293HA. Yellow triangles denote the hybrid plasmids. Strains with the same symbol on the PFGE image represent progeny derived from the same parental strain. $\mathbf{b}$ Schematic diagram of the conjugation assays. The red square denotes the hygromycin B resistance gene $h p h$ tag on the virulence plasmid pRJF293HA. The green rectangle denotes the tra gene cluster encoding a T4SS on three IncF plasmids derived from RJBSI76, pRJBSI76-1 (p1), pRJBSI76-2 (p2) and pRJBSI76-3 (p3). The plasmid p1 in transconjugant RJBSI76-pV became smaller ( $\mathrm{p}^{\prime}$ ). Full details of the abovementioned plasmids are shown in Additional file 2: Figs. S1-S5

inhibit plasmid transmission [37-39], the donor was switched to the reduced extracellular polysaccharide mutant rmpA-deficient RJF293HA (RJF293 $\Delta r m p A:: h p h$ ) for the following three rounds of conjugation (Fig. 1).

In the first round, the nonconjugative virulence plasmid pRJF293HA (also represented as $\mathrm{pV}$ in this study) was only transferred into CRKP RJBSI76. The obtained virulence plasmid pRJF293HA in the transconjugants RJBSI76-pV and RJBSI76-pV-hybrid showed two different patterns: (i) kept as a single plasmid and (ii) fused with pRJBSI76-1 (represented as p1) to yield a hybrid plasmid (named p1-pV-hybrid-1) (Fig. 1; Table 1). The virulence plasmids in both patterns were stable after serial cultures (Additional file 2: Fig. S1). The WGS of the transconjugant RJBSI76-pV suggested that the shorter plasmid pRJBSI76-pV-1 (represented as p1') might be the result of two crossover homologous recombination events between pRJBSI76-1 and pRJF293HA (Additional file 2: Fig. S2).

In the second round, pRJF293HA could be further transferred from transconjugant RJBSI76-pV to E. coli J53 accompanied or not accompanied by the shorter plasmid p1'. The hybrid plasmid p1-pV-hybrid-1 in the transconjugant RJBSI76-pV-hybrid was transferred as a cointegrate. Similar findings were also observed in the third round (Fig. 1; Table 1). Notably, in the absence of

Table 1 Conjugation frequency of the virulence plasmid pRJF293HA and its derivatives

\begin{tabular}{|c|c|c|c|}
\hline Transconjugant & Donor & Recipient & Conjugation frequency \\
\hline RJBSI76-pV (iroB $\left.{ }^{+}\right)$ & RJF293HA (pV-harbouring, iro $B^{+}$) & RJBSI76 (p1-harbouring) & $(1.01 \pm 0.30) \times 10^{-7}$ \\
\hline RJBSI76-pVC (iro $\left.B^{+}, r m p A^{+}\right)$ & C600-p1'-pVC (iro $\left.B^{+}, r m p A^{+}\right)$ & RJBSI76 & $(5.59 \pm 2.79) \times 10^{-6}$ \\
\hline $\mathrm{J} 53-\mathrm{pV}^{\mathrm{b}}\left(\right.$ iro $\left.B^{+}\right)$ & RJBSI76-pV (iro $\left.B^{+}\right)$ & J53 & $(1.43 \pm 0.74) \times 10^{-4}$ \\
\hline J53-pVC $\left(i r o B^{+}, r m p A^{+}\right)$ & RJBSI76-pVC (iro $\left.B^{+}, r m p A^{+}\right)$ & $\mathrm{J} 53$ & $(3.03 \pm 1.46) \times 10^{-7}$ \\
\hline J53-p1-pV-hybrid-1 (iroB ${ }^{+}$) & RJBSI76-pV-hybrid (iro ${ }^{+}$) & J53 & $(4.58 \pm 1.48) \times 10^{-5}$ \\
\hline C600-p1-pV-hybrid (iroB ${ }^{+}$) & J53-p1-pV-hybrid-1 (iroB $\left.{ }^{+}\right)$ & C600 & $(2.02 \pm 0.22) \times 10^{-1}$ \\
\hline C600-pV $V^{d}\left(\right.$ iro $\left.B^{+}\right)$ & J53-p1'-pV-TC1 (iroB $\left.{ }^{+}\right)$ & C600 & $(4.18 \pm 1.47) \times 10^{-2}$ \\
\hline J53-p1 & RJBSI76 & 553 & $(2.38 \pm 0.50) \times 10^{-3}$ \\
\hline J53-p1'-pV-TC2 $\left(i r o B^{+}\right)$ & RJF293HA (pV-harbouring, iro $B^{+}$) & J53-p1 & $(2.22 \pm 0.45) \times 10^{-7}$ \\
\hline C600-p1 & $J 53-p 1$ & C600 & $(1.88 \pm 0.56) \times 10^{-2}$ \\
\hline C600-p1'-pV-TC2 (iroB $\left.{ }^{+}\right)$ & RJF293HA (pV-harbouring, iro $B^{+}$) & C600-p1 & $(5.53 \pm 0.63) \times 10^{-6}$ \\
\hline C600-p1'-pVC (iro $\left.B^{+}, r m p A^{+}\right)$ & RJF293C (pVC-harbouring, iro $B^{+}, r m p A^{+}$) & C600-p1 & $(5.83 \pm 3.49) \times 10^{-8}$ \\
\hline J53-pF-pV-TC1 (iro $\left.B^{+}\right)$ & XL10-pF & J53-pV (iroB $\left.{ }^{+}\right)$ & $(4.19 \pm 0.21) \times 10^{-2}$ \\
\hline XL10-pF-pV-TC1 ${ }^{f}\left(\right.$ iro $\left.B^{+}\right)$ & J53-pV $\left(\right.$iro $\left.B^{+}\right)$ & XL10-pF & $(1.04 \pm 0.57) \times 10^{-7}$ \\
\hline 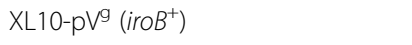 & J53-pF-pV-TC1 (iroB $B^{+}$ & XL10 & $(2.65 \pm 1.33) \times 10^{-6}$ \\
\hline XL10-pF-TC & J53-pF-pV-TC1 (iro $\left.B^{+}\right)$ & $X L 10$ & $(2.71 \pm 1.04) \times 10^{-3}$ \\
\hline J53-pF-pV-TC2 ${ }^{h}\left(\right.$ iro $\left.^{+}\right)$ & XL10-pF-pV-hybrid-1 (iro $\left.B^{+}\right)$ & $\mathrm{J} 53$ & $(6.74 \pm 0.72) \times 10^{-2}$ \\
\hline$J 53-p V_{W T}^{i}\left(i r o B^{+}, r m p A^{+}\right)$ & RJF293-pF ( $p V_{W T}$-harbouring, iro $B^{+}, r m p A^{+}$) & $J 53$ & $(2.04 \pm 0.36) \times 10^{-6}$ \\
\hline HS11286-pF-pV $V_{W T}-^{j}\left(\right.$ iroB $\left.^{+}, r m p A^{+}\right)$ & J53-pF-pV $V_{W T}-h y b r i d$ & HS11286-pKPHS2 $\Delta$ oriT & $(1.46 \pm 0.21) \times 10^{-5}$ \\
\hline HS11286-pF-pV $V_{W T}-2^{k}\left(\right.$ iro $\left.B^{+}, r m p A^{+}\right)$ & J53-pF-pV $V_{W T}$ & HS11286-pKPHS2 $\triangle$ oriT & $(1.68 \pm 0.28) \times 10^{-9}$ \\
\hline J53-pF $\Delta$ oriT-p1 $1^{1}$ & XL10-pF $\Delta$ oriT-p1 & $\mathrm{J} 53$ & $(4.04 \pm 0.52) \times 10^{-7}$ \\
\hline
\end{tabular}

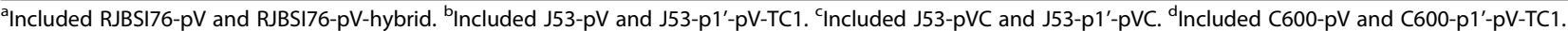
eIncluded J53-p1'-pV-TC2 and J53-p1-pV-hybrid-2. 'Included XL10-pF-pV-TC1 and XL10-pF-pV-hybrid-1. ' Included XL10-pV, XL10-pF-pV-TC2 and XL10-pF-pV-hybrid-

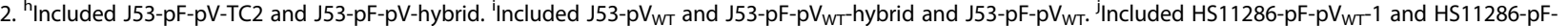

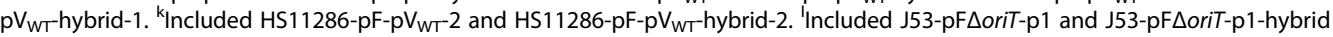


$\mathrm{p} 1$ ', the virulence plasmid in transconjugant J53-pV could not be transferred (Fig. 1). The WGS of the transconjugant J53-p1-pV-hybrid-1 suggested that the hybrid plasmid was a recombinant of pRJF293HA and pRJBSI76-1 with a 22-kb DNA fragment of pRJF293HA containing $h p h$ and iroBCDN inserted into the backbone of the IncF plasmid pRJBSI76-1 (Additional file 2: Fig. S3).

Overall, the nonconjugative virulence plasmid could be transferred from hvKP to ST11 CRKP and further to E. coli with or without the formation of the hybrid plasmid that emerged from homologous recombination.

\section{Mobilization of the pLVPK-like virulence plasmid with the} help of the self-transferable IncF plasmid pRJBSI76-1

According to the WGS analysis, RJBSI76 contains three IncF plasmids and one small ColRNAI plasmid. The IncFIB plasmid pRJBSI76-1 (p1) carries known virulence genes (rmpA2, iucABCDiutA) and tellurite resistance genes (ter $Z A B C D E$ and ter $W$ ) (Additional file 2: Fig. S4). The IncFII $_{K} /$ IncFIB $_{K}$ plasmid pRJBSI76-2 (p2) encodes resistance to various classes of antibiotics (Additional file 2: Fig. S5a). The IncFII ${ }_{\mathrm{pHN7A}} / \mathrm{IncR}$ plasmid pRJBSI76-3 (p3) carries $b l a_{\mathrm{KPC}-2}$ and other antimicrobial resistance genes (Additional file 2: Fig. S5b). Notably, all three IncF plasmids carry the tra gene clusters that encode the conjugative apparatus (Additional file 2: Fig. S4-S5).

To investigate which of the IncF plasmids of RJBSI76 plays a critical role in helping the transfer of pRJF293HA, we first evaluated the conjugative ability of three plasmids. Only pRJBSI76-1 was successfully transferred from RJBSI76 to J53, and the acquired pRJBSI76-1 helped the transmission of pRJF293HA from RJF293HA to J53-p1 with or without hybrid plasmid formation. Similarly, E. coli C600-p1 gained the virulence plasmid when conjugated with RJF293HA (Fig. 2; Table 1). These results showed that pRJBSI76-1 does play a critical role in the mobilization of virulence plasmid.

We also explored whether the conjugative transfer regions of the IncF plasmid pRJBSI76-1, including the tra genes and the oriT region, are involved in virulence plasmid mobilization [40]. We constructed a mimic virulence plasmid carrying the predicted oriT region of pRJF293HA, which was denoted pACYC184-Aprori $_{\mathrm{RJF293HA}}$. The nucleotide sequence of the oriT regions of pRJF293HA and pRJBSI76-1 showed 100\% identities (Additional file 2: Fig. S6). The empty vector pACYC184-Apr was nonconjugative but pACYC184Apr-oriT $T_{\mathrm{RJF} 293 \mathrm{HA}}$ was transferred from C600-p1ori $_{\text {RJF293HA }}$ to J53 (Fig. 3a), which suggested that pRJBSI76-1 could mobilize the nonconjugative mimic virulence plasmid containing the oriT of pRJF293HA. We then knocked out the traE gene that encodes an essential component of T4SS responsible for seeding the site of pilus assembly [41] or the predicted oriT region on pRJBSI76-1 in E. coli C600-p1. traE-deficient pRJBSI76-1 failed to mobilize pACYC184-AproriT $_{\text {RJF293HA }}$ (Fig. 3b). Interestingly, pACYC184-Aprori $T_{\mathrm{RJF} 293 \mathrm{HA}}$ could also transfer alone with the help of oriT-deficient pRJBSI76-1 at a very low frequency (Fig. $3 \mathrm{~b})$, which indicated the rolling circle replication of pACYC184-Apr-ori $T_{\text {RJF293HA, and the subsequent trans- }}$ fer could be finished with the help of the conjugative apparatus of pRJBSI76-1, which was consistent with the previously reported mobilization utilizing the T4SS on the helper plasmid [42]. In addition, neither modified pRJBSI76-1 nor pRJF293HA was transferred in the conjugation between RJF293HA and C600-p1 1 traE or C600-p1 $\Delta$ oriT (Fig. 3c).

The above data showed that the nonconjugative virulence plasmid pRJF293HA could be mobilized by the self-transferable IncF plasmid pRJBSI76-1 encoding a functional T4SS.

\section{Mobilization of the pLVPK-like virulence plasmid with the help of the conjugative IncF plasmid pOX38-Gen}

Because the large IncF plasmid pRJBSI76-1 has a complicated structure, we employed the IncFIA plasmid derivative pOX38-Gen (also represented as pF) to further investigate the mechanism of pRJF293HA mobilization. The transmissions of both pOX38-Gen and pRJF293HA were observed in the conjugation between XL10-pF and J53-pV. Remarkably, two types of transconjugants contained virulence plasmids: pRJF293HA was fused with pOX38-Gen or remained unchanged (Fig. 4; Table 1).

In the conjugation between XL10 and J53-pF-pV-TC1 harbouring separate pOX38-Gen and pRJF293HA, various modes of pRJF293HA mobilization were detected: (i) fused with pOX38-Gen, (ii) transferred with pOX38Gen and (iii) transferred alone. The hybrid plasmid of XL10-pF-pV-hybrid-1 could further be transferred to J53, which yielded transconjugants with or without hybrid plasmid resolution (Fig. 4; Table 1). The formation and resolution of the hybrid plasmid pF-pV-hybrid-1 were observed during serial passages (Additional file 2: Fig. S7), which suggested that the fusion event was reversible. A WGS analysis of XL10-pF-pV-hybrid-1 revealed that pRJF293HA was entirely integrated into pOX38-Gen between 28-bp fusion sites shared on both plasmids (Fig. 5). The results indicate that the specific regions consist of two conserved 10-bp inverted repeat sequences and a variable 8-bp internal spacer region where the recombinant junction was supposed to occur. It has been proposed that two rounds of strand cleavage and exchange lead to the generation and resolution of a Holliday junction intermediate [43]. To validate the specific fusion event, conjugation between XL10-pF $\Delta$ oriTp1 and J53 was performed. The nonconjugative oriT- 


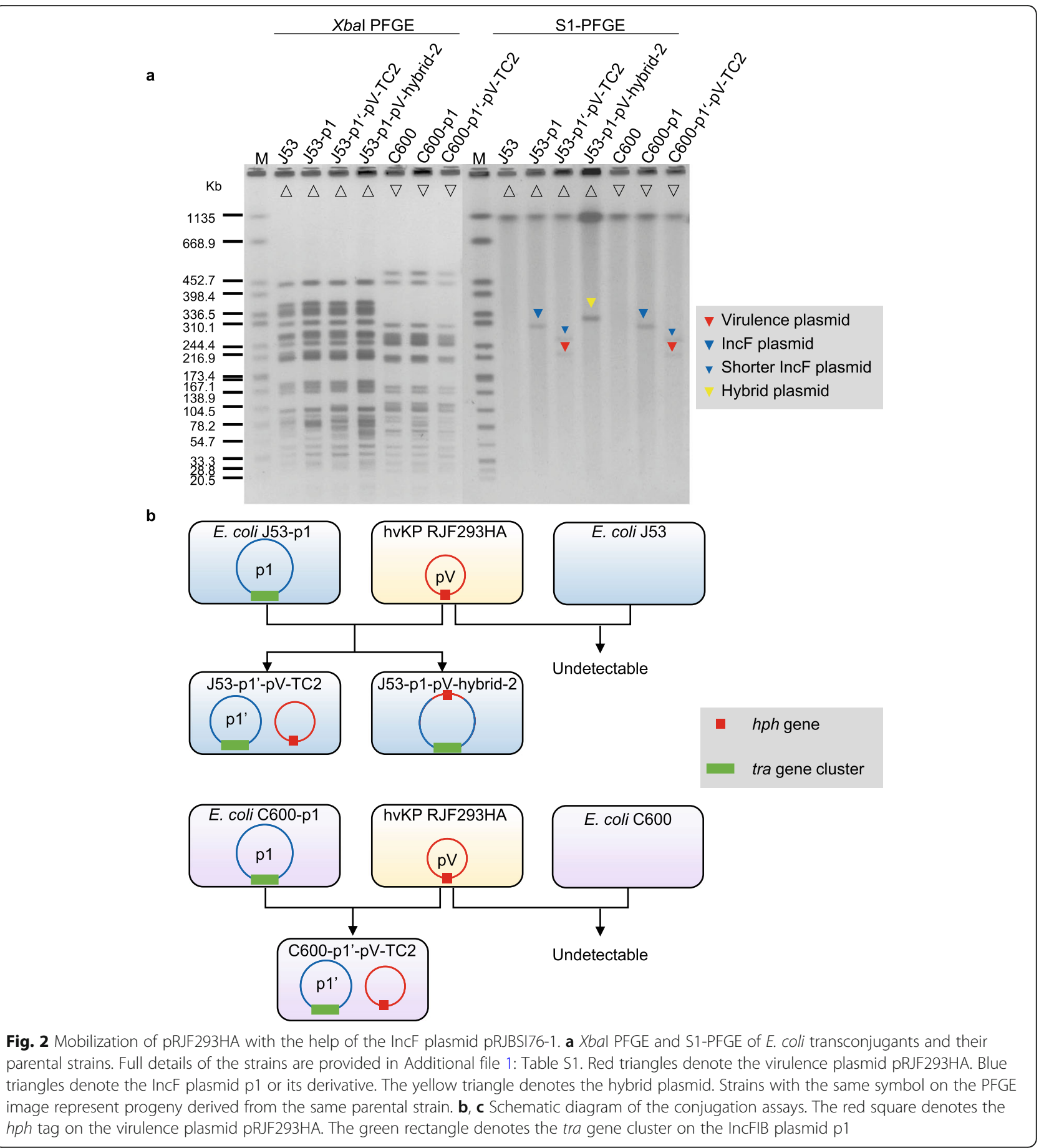

deficient pOX38-Gen was mobilized by pRJBSI76-1 with or without a fusion event at 28-bp fusion sites (Additional file 2: Fig. S8).

To further investigate the mechanism underlying mobilization without hybrid plasmid formation, the mimic virulence plasmid and knockout strains were employed. The mimic virulence plasmid pACYC184Apr-ori $T_{\text {RIF293HA }}$ rather than the empty vector pACYC184-Apr could be mobilized by pOX38-Gen
(Additional file 2: Fig. S9a). Both oriT-deficient pOX38Gen and traE-deficient pOX38-Gen lost their conjugative ability and the transmission of pACYC184-Aprori $_{\text {RIF293HA }}$ or the virulence plasmid pRJF293HA was not detected (Additional file 2: Fig. S9b, c).

Altogether, these results showed that the nonconjugative virulence plasmid pRJF293HA containing oriT and the 28-bp fusion site could transfer alone, cotransfer with the IncF plasmid or fuse with the IncF plasmid at 

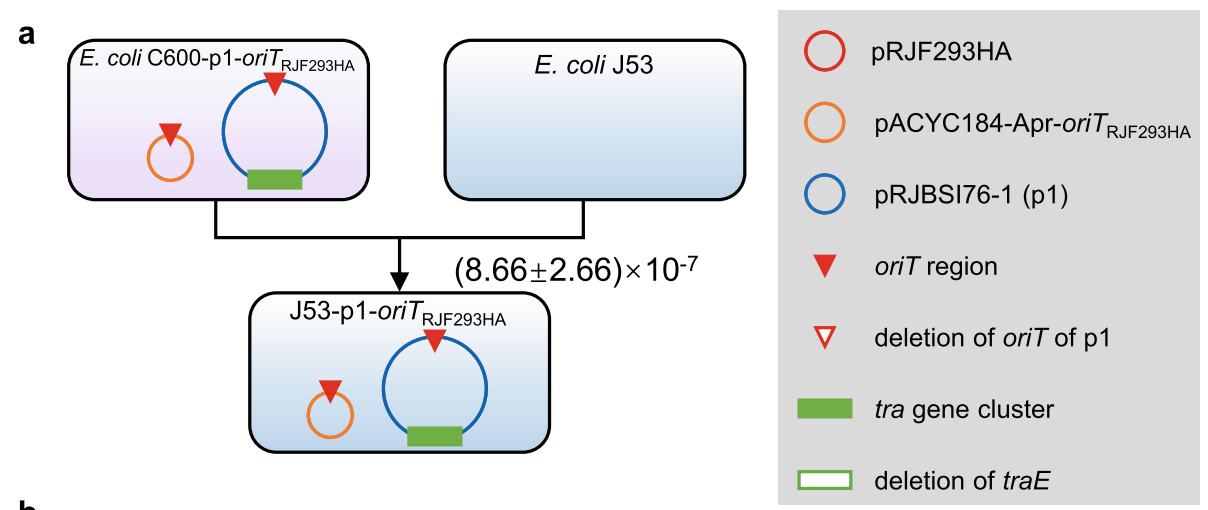

b

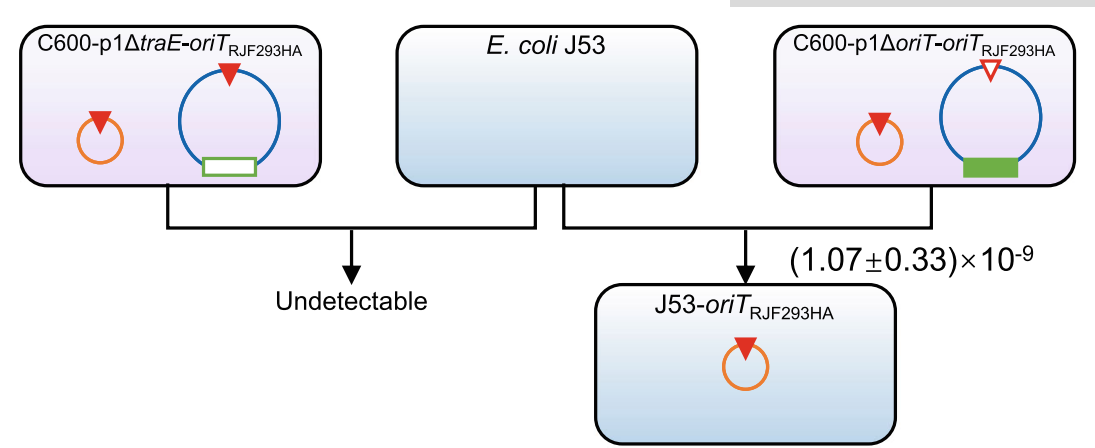

C

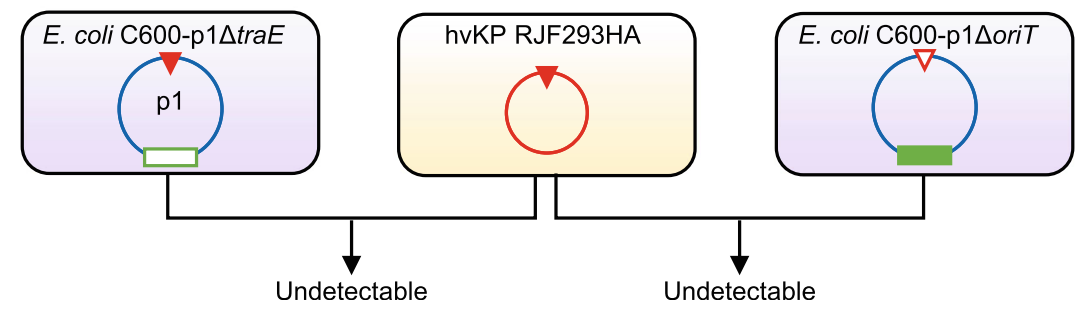

Fig. 3 Schematic diagram of conjugation assays for the IncF plasmid pRJBSI76-1 and its nonconjugative derivatives. The red circle denotes the

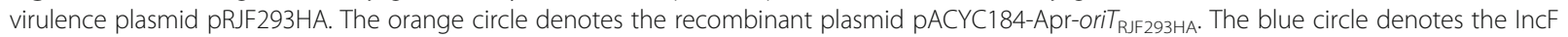
plasmid pRJBSI76-1 (p1). The red triangle denotes the oriT region of pRJF293HA (oriT RJF293HA) or that of p1. The green rectangle denotes the tra gene cluster on the IncF plasmid p1. The hollow red triangle on $\mathrm{p} 1$ represents the deletion of the oriT region. The hollow green rectangle on $\mathrm{p} 1$ indicates that the gene traE was deleted

28-bp fusion sites with the help of the IncF plasmid pOX38-Gen harbouring a functional T4SS.

\section{E. coli promoted indirect virulence plasmid transfer from hvKP with overproduced extracellular polysaccharides into CRKP}

The rmpA-positive virulence plasmid pRJF293C (represented as pVC) was not transferred between RJF293C and RJBSI76 but was successfully transferred from RJF293C to C600-p1 and further to RJBSI76, leaving the K. pneumoniae transconjugant RJBSI76-pVC positive for the string test (Table 1; Additional file 2: Fig. S10). Compared with RJF293HA and RJBSI76-pV, RJF293C and RJBSI76-pVC less easily transferred the virulence plasmid to the E. coli strain, and these difficulties might be explained by significant increases in mucoviscosity and extracellular polysaccharide production (Additional file 2: Fig. S11).

Similarly, the virulence plasmid pRJF293 (also represented as $\mathrm{pV}_{\mathrm{WT}}$ ) was not transferred between RJF293-pF and HS11286 or its derivative HS11286-pKPHS2 $\triangle$ oriT, whereas $E$. coli 553 could serve as an intermediate vector to promote virulence plasmid transfer. pRJF293 was transferred from RJF293-pF to E. coli 553 alone or together with pOX38-gen with or without hybrid plasmid formation. The virulence plasmid was transferred from J53-pF-pV $V_{\mathrm{WT}}$ and J53-pF-pV $\mathrm{WT}_{\mathrm{WT}}$-hybrid to HS11286pKPHS2 2 oriT via various modes, and significantly higher conjugation frequency was obtained with hybrid plasmid formation in the donor strain $[(1.46 \pm 0.21) \times$ $10^{-5}$ versus $(1.68 \pm 0.28) \times 10^{-9}, P=0.00058$ ] (Table 1 ; Additional file 2: Fig. S12). The acquisition of pRJF293 


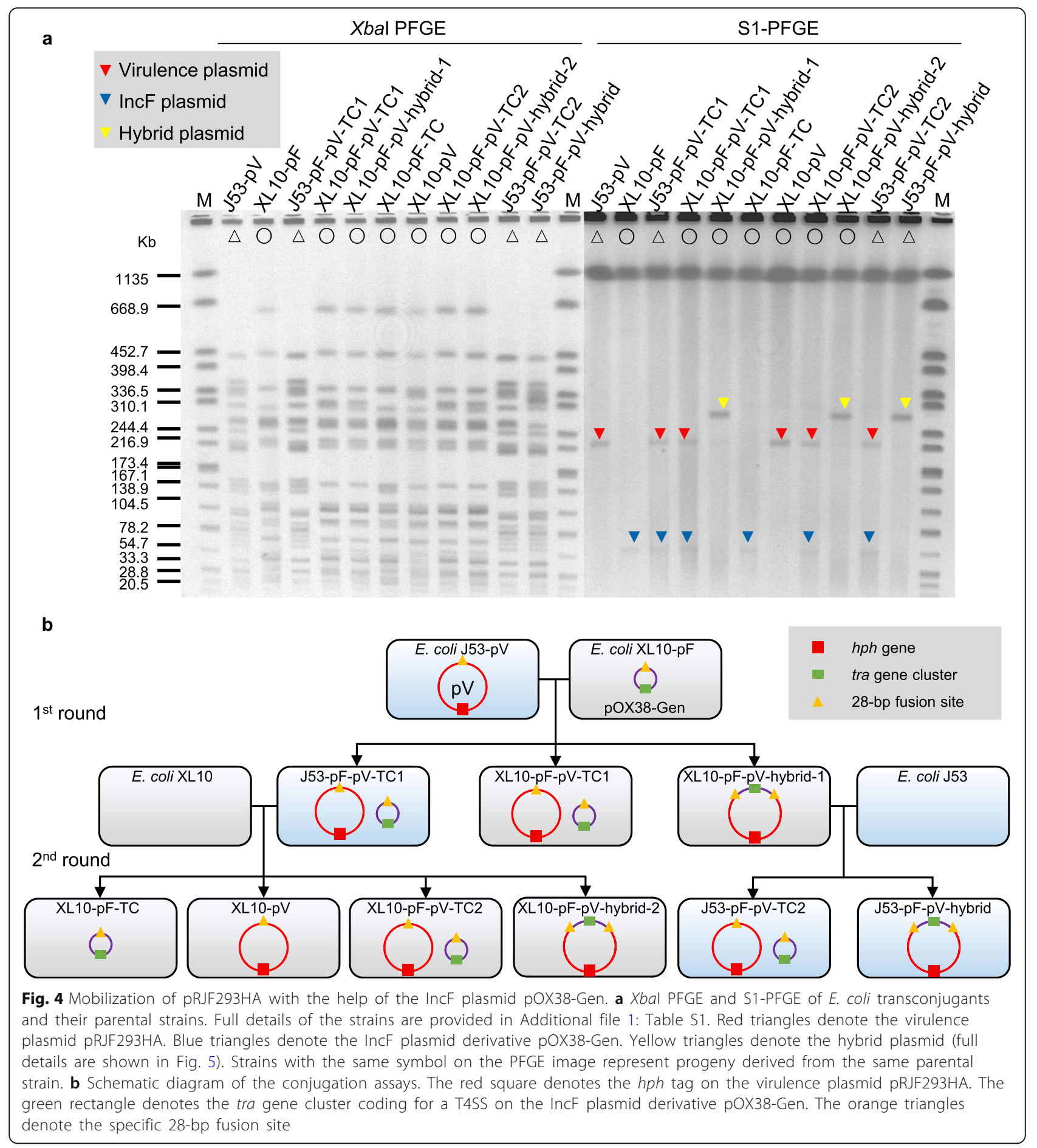

also made HS11286-pKPHS2 $\Delta$ oriT transconjugants hypermucoviscous.

The above-described results showed that the virulence plasmid could be transferred from conjugative IncF plasmid-containing hvKP to E. coli. The E. coli transconjugants containing both virulence and IncF plasmids, with or without hybrid plasmid formation, could further transfer the virulence plasmid or the hybrid plasmid to CRKP via different modes.

\section{Distribution of putative helper plasmids and mobilizable virulence plasmids among $K$. pneumoniae}

Based on the above-described conjugation results and the previously reported requirements for helper plasmids 


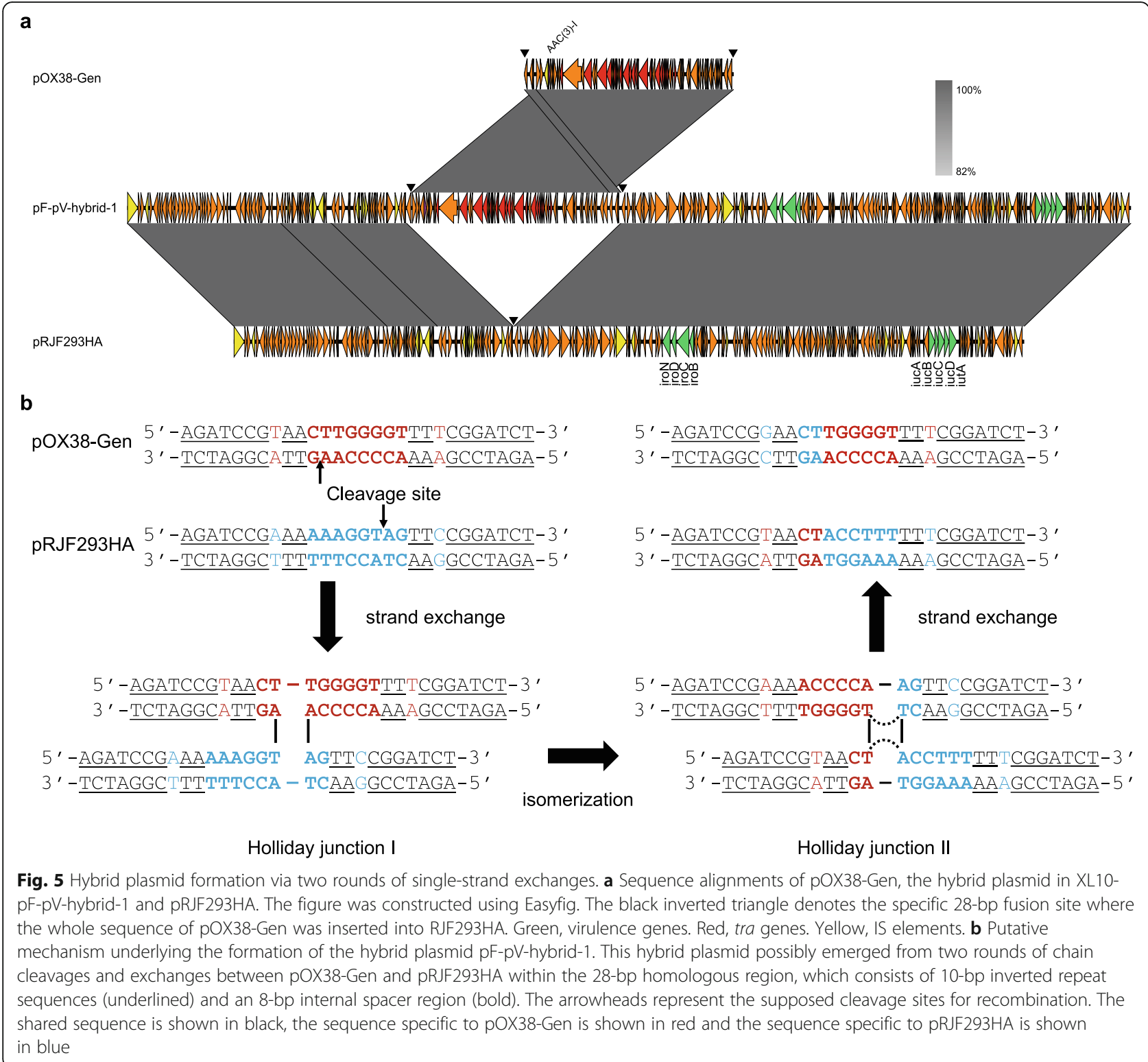

[40], we hypothesized that conjugative plasmids are potential helper plasmids in K. pneumoniae and that virulence plasmids containing the oriT region or the specific 28-bp fusion site are mobilizable. We searched 2608 plasmids in 814 completely sequenced $K$. pneumoniae strains available in GenBank to investigate the distribution of putative helper plasmids and mobilizable virulence plasmids.

A total of $29.1 \%(760 / 2608)$ of plasmids are considered conjugative and have the ability to mobilize virulence plasmids containing the oriT region (Additional file 2: Fig. S13), and $31.8 \%$ (242) of these plasmids were found to carry the 28-bp fusion site and might have the potential to mobilize virulence plasmids containing the 28-bp fusion site, resulting in the formation of hybrid plasmids. A total of 2.2\% (17) of the putative helper plasmids carry two or three 28-bp fusion sites, whose significance remains to be investigated. The putative conjugative plasmids exhibit a wide distribution range over the $K$. pneumoniae strains of various sequence types, and ST11 is the dominant clone type, followed by ST258, ST147, ST231 and others. Notably, $67.6 \%$ (514) of the putative helper plasmids were IncF plasmids and showed a higher percentage of plasmids containing 28-bp fusion sites than non-IncF-type plasmids $(47.8 \%$ vs $5.7 \%$ ). Significantly, $75.8 \%$ (576) of the predicted conjugative plasmids contained one or more antimicrobial resistance genes, and 23.8\% (181) harboured carbapenemase genes, which suggested that $K$. pneumoniae 
strains with multidrug resistance and hypervirulence could potentially be generated by cotransfer of the virulence plasmids with the IncF plasmids encoding antimicrobial resistance.

A total of $85 \mathrm{~K}$. pneumoniae virulence plasmids were extracted (Additional file 2: Fig. S14). Six virulence plasmids harbour at least one antimicrobial resistance gene. Six plasmids contain T4SS gene clusters, and the virulence plasmids were distributed in both cKP clone types (e.g. ST11, ST15 and ST383) and hvKP clone types (e.g. ST23, ST65, ST66 and ST86). All the virulence plasmids harbour the oriT region, and 84 of them have at least a specific 28-bp fusion site, which indicates that all the selected virulence plasmids can be considered mobilizable.

\section{Discussion}

The pLVPK-like virulence plasmid of hvKP is generally considered to be nonconjugative and absent from cKP. Several studies conducted in recent years have reported emerging virulence plasmid-harbouring cKP strains [2, $8,9]$, which has raised public concerns regarding the availability of an effective treatment for this type of threatening pathogen. However, the process of virulence plasmid transfer from hvKP to CRKP and the important elements essential for virulence plasmid transfer remain unclarified. Here, we demonstrated that under reduced extracellular polysaccharide-producing conditions or by employing $E$. coli intermediate strains, the pLVPK-like nonconjugative virulence plasmid could be transferred from hvKP to ST11 CRKP strains via four different modes (Fig. 6). The IncF plasmid, as a driving force in virulence plasmid mobilization, is reportedly prevalent in
Enterobacteriaceae worldwide and carries a great variety of antimicrobial resistance genes, accounting for almost $40 \%$ of plasmid-borne carbapenemases [44, 45]. Attention should be given to the potential risks that antibiotic selection might also promote the dissemination of virulence plasmids and enrich hypervirulent multidrugresistant cKP (hv-MDR-cKP) or hv-CRKP strains.

The virulence plasmid pRJF293HA in the presence of oriT was found to be transferred alone or cotransferred with the conjugative IncF plasmid. The reported virulence plasmids in ST11 or ST15 cKP strains might be obtained through the former two approaches $[9,17,46]$. The oriT sequence of the previously submitted virulence plasmid p17-16-vir was the same as that of pRJF293, and p17-16-vir was found to be transferred via mode $i$ and mode iv [17] (Fig. 6). A functional T4SS is an indispensable element for helper plasmids, as has been verified by the loss of self-transferability and failure in the mobilization of virulence plasmids or mimic virulence plasmids after the deletion of traE on IncF plasmids. We also noticed that the helper plasmid could employ the oriT of the mobilized plasmid to complete the mobilization, which was consistent with a previous study [42].

The previously reported hybrid plasmids include those that emerged from homologous recombination in $K$. pneumoniae strains [16] and IS26-mediated incorporation in a Salmonella enteritidis strain [47]. Here, we identified a novel fusion event between a nonconjugative virulence plasmid and a self-transferable IncF plasmid due to site-specific recombination: mode iii. The specific 28-bp fusion site of pOX38-Gen was previously reported

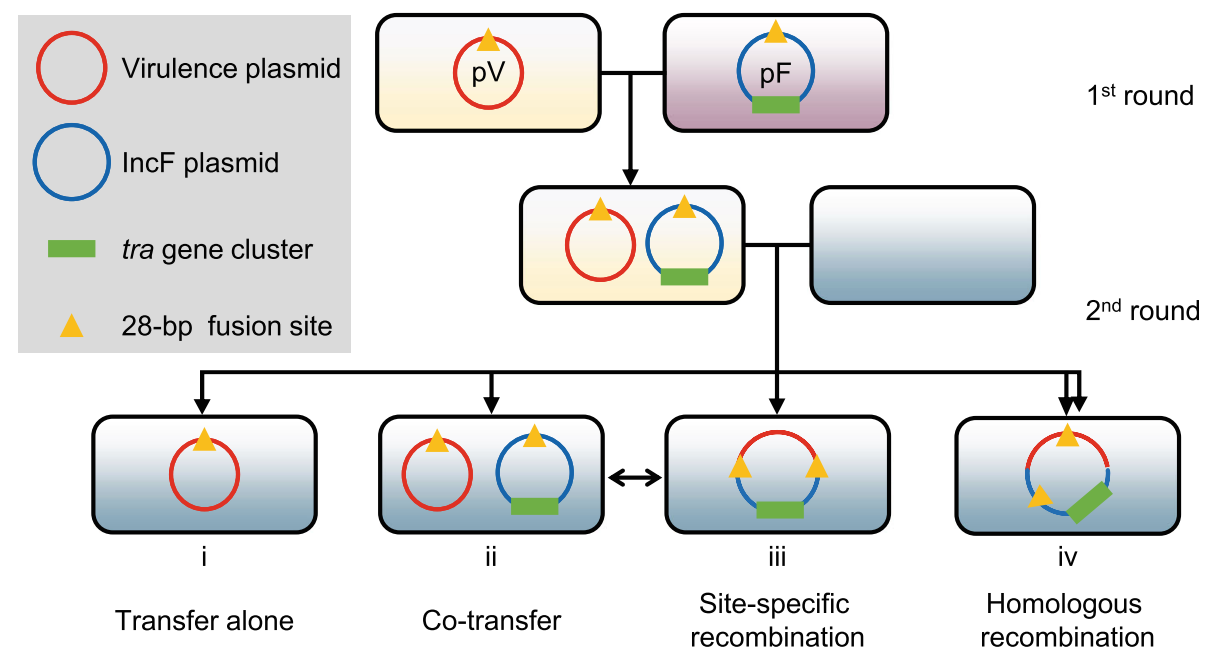

Fig. 6 Proposed model of the mobilization of the pLVPK-like nonconjugative virulence plasmid of $K$. pneumoniae. The initial step of virulence plasmid transfer is the acquisition of a conjugative IncF plasmid, and this step is followed by virulence plasmid mobilization via four modes. The virulence plasmid could be transferred (i) alone, (ii) cotransferred with the IncF plasmid, (iii) fused with the IncF plasmid due to recombination at specific 28-bp fusion sites or (iv) fused with the IncF plasmid due to recombination in the homologous region. The green rectangle denotes the tra gene cluster coding for a T4SS on the IncF plasmid. The orange triangles denote the specific 28-bp fusion site 
and named the replicon fusion site of $\mathrm{F}(r f s F)$ [43], within which recombination and resolution events were observed only between $\mathrm{F}$ plasmids via two rounds of single-strand exchanges [48]. Similarly, integration and resolution events occurred between the virulence plasmid pRJF293HA and the IncF plasmid pOX38-Gen at the specific 28 -bp fusion sites. This fusion event was repeated between pRJBSI76-1 and pOX38-Gen $\Delta$ oriT (Additional file 2: Fig. S8). A more efficient virulence plasmid transfer was observed after hybrid plasmid formation at 28-bp fusion sites in the donor strain (Table 1; Additional file 2: Fig. S12), which might allow the mobilization of virulence plasmids by a broader set of IncF plasmids. Whether the various modes proposed in our study are universal in clinical settings and whether other mobilization modes exist require further largescale surveillance studies on virulence plasmid transmission and evolution.

According to the in silico analysis of the completely sequenced K. pneumoniae available in GenBank, 29.1\% of plasmids possess a conjugative apparatus and have the potential to serve as helper plasmids. We also found that all the virulence plasmids were mobilizable because they contained an oriT region with or without 28 -bp fusion site(s). The 28-bp fusion sites were also distributed in $31.8 \%$ of the helper plasmids. These results alarmingly indicate that antibiotic abuse might contribute to the explosive dissemination of helper plasmids coding for antimicrobial resistance $[49,50]$ and promote the transfer of virulence plasmids and the emergence of hv-MDR-cKP or hv-CRKP.

The virulence plasmid was not transferred in the direct interactions between hypermucoviscous hvKP RJF293C and ST11 CRKP strains but was mobilized from rmpA-deficient mutant RJF293HA to CRKP RJBSI76. In addition, the interspecies transmission of pRJF293C was less frequent than that of pRJF293HA. Our findings are consistent with previous studies that reported that overproduced extracellular polysaccharides might serve as a barrier for plasmid transfer because they could conceal crucial attachment sites for the conjugative apparatuses, such as OmpA porin and lipopolysaccharide [37, 51, 52]. E. coli strains harbouring conjugative IncF plasmids could serve as the intermediate vector to deliver the virulence plasmid indirectly from hypermucoviscous hvKP into CRKP, resulting in the transconjugants hypermucoviscosity phenotype, which is usually associated with hypervirulence $[4,53]$. The results imply that the microbial environment might influence virulence plasmid transmission by regulating extracellular polysaccharide production, and E. coli strains might also serve as a reservoir of the virulence plasmid of K. pneumoniae.

\section{Conclusions}

Our study first confirmed the mobilization of a pLVPKlike nonconjugative virulence plasmid from hvKP into CRKP and E. coli strains with the help of a selftransferable IncF plasmid. The various mobilization modes observed in the study deepened our understanding of the transfer of virulence plasmids. Inappropriate antibiotic usage might boost the transfer of conjugative plasmids encoding antimicrobial resistance and the dissemination of nonconjugative virulence plasmids, which contributes to the ongoing emergence of $K$. pneumoniae with both antimicrobial resistance and hypervirulence.

\section{Abbreviations}

CFU: Colony-forming unit; cKP: Classic Klebsiella pneumoniae; CRhvKP: Carbapenem-resistant hypervirulent Klebsiella pneumoniae; CRKP: Carbapenem-resistant Klebsiella pneumoniae; FRT: Flippase recognition sites; hv-CRKP: Hypervirulent carbapenem-resistant Klebsiella pneumoniae; hvMDR-cKP: Hypervirulent multidrug-resistant classic Klebsiella pneumoniae; hvKP: Hypervirulent Klebsiella pneumoniae; IncF: Incompatibility group F; IR: Inverted repeat; IS: Insertion sequence; LB: Lysogeny broth; NCBI: National Center for Biotechnology Information; nic: Nick site at the origin of transfer (oriT); oriT: Origin of transfer; PFGE: Pulsed-field gel electrophoresis; rfsF: Replicon fusion site of F; SD: Standard deviation; ST: Sequence type based on the multilocus sequence typing; T4CP: Type IV coupling protein; T4SS: Type IV secretion system; WGS: Whole-genome sequencing

\section{Supplementary Information}

The online version contains supplementary material available at https://doi. org/10.1186/s13073-021-00936-5.

Additional file 1. Table S1. Strains and plasmids used in this study.

Additional file 2. Supplementary Methods. Figure S1. Plasmid stability of serial cultures of the K. pneumoniae transconjugants RJBSI76-pV and RJBSI76-pV-hybrid. Figure S2. Homologous recombination between the IncF plasmid pRJBSI76-1 and the virulence plasmid pRJF293HA. Figure S3. Sequence alignments of the IncF plasmid pRJBSI76-1, the hybrid plasmid p1-pV-hybrid-1 and the virulence plasmid pRJF293HA. Figure S4. Genetic structure of the IncFIB plasmid pRJBSI76-1 of the clinical CRKP strain RJBSI76. Figure S5. Genetic structure of the plasmids pRJBSI76-2 and pRJBSI76-3 of the clinical CRKP strain RJBSI76. Figure S6. Sequence alignment of three predicted oriT regions. Figure S7. Plasmid stability of serial cultures of the E. coli transconjugants J53-pF-pV-hybrid and J53-pF-pVTC2. Figure S8. Validation of the fusion event at 28-bp fusion sites. Figure 59. Schematic diagram of the mobilization of a virulence plasmid by the conjugative IncF plasmid pOX38-Gen. Figure S10. Indirect transfer of the virulence plasmid from hvKP RJF293C to CRKP RJBSI76. Figure S11. The less-frequent transfer of pRJF293C was due to increased production of extracellular polysaccharides by the donor. Figure S12. Indirect transfer of the virulence plasmid from hvKP RJF293-pF to CRKP HS11286-

PKPHS2 2 oriT. Figure S13. In silico analysis of 760 conjugative plasmids of the completely sequenced K. pneumoniae in GenBank. Figure S14. Mobilization potential of 85 virulence plasmids of the completely sequenced K. pneumoniae available in GenBank.

Additional file 3. Table S2. Oligonucleotides used in this study.

Additional file 4. Table S3. Antibiotics and corresponding concentrations used for each conjugation pair.

Additional file 5. Table S4. NCBI accession numbers and genetic characteristics of 2608 K. pneumoniae plasmids.

Additional file 6. Table S5. NCBI accession numbers and genetic characteristics of 760 putative conjugative plasmids of K. pneumoniae.

Additional file 7. Table S6. NCBI accession numbers and genetic characteristics of 85 putative mobilizable virulence plasmids of $K$. pneumoniae. 


\section{Acknowledgements}

We thank Professor Xiaofei Jiang for providing pOX38-Gen containing strain, named as CW51-pF.

\section{Authors' contributions}

Y.X. designed the study, performed experiments and revised the manuscript. J.Z. constructed the gene knockout strains and revised the manuscript. M.W. assembled and analysed the whole-genome sequencing data. M.L. predicted the oriT sequence. G. L participated in experiment performance. H.Q, J.L and Z.D. provided strains. Y.X, H.Y.O and J.Z. drafted the manuscript. H.Y.O., J.Q. and S.J. designed the study, supervised the whole project and revised the manuscript. All authors read and approved the final manuscript.

\section{Funding}

National Key Research and Development Program of China (2018YFE0102400); Science and Technology Commission of Shanghai Municipality (19JC1413000 and 19430750600); Cultivation Project of Shanghai Major Infectious Disease Research Base (20dz2210500); Key Laboratory of Emergency Prevention, Diagnosis and Treatment of Respiratory Infectious Diseases, Shanghai (20dz2261100); Medicine and Engineering Interdisciplinary Research Fund of Shanghai Jiao Tong University (19X190020171); National Innovative Research Team of High-level Local Universities in Shanghai. The funding sources had no influence on the design of the study; collection, analysis and interpretation of the data; and writing of the manuscript.

\section{Availability of data and materials}

The complete genome sequences of K. pneumoniae RJBSI76 and RJBSI76-pV, as well as E. coli J53-p1-pV-hybrid-1, were deposited in the NCBI BioProject repository under the accession numbers PRJNA681750 (https://www.ncbi. nlm.nih.gov/nuccore/?term=PRJNA681750) [22], PRJNA682095 (https://www. ncbi.nlm.nih.gov/nuccore/?term=PRJNA682095) [23], and PRJNA692573 (https://www.ncbi.nlm.nih.gov/nuccore/?term=PRJNA692573) [24]. The genome sequence of the hybrid plasmid pF-pV-hybrid-1 derived from E. coli strain XL10-pF-pV-hybrid-1 was deposited in the NCBI BioProject repository under the accession number PRJNA692574 (https://www.ncbi.nIm.nih.gov/ nuccore/1968731931) [25]. The accession numbers of all the other sequences analysed during the current study are included in this manuscript and available in the NCBI Nucleotide database [54].

\section{Declarations}

\section{Ethics approval and consent to participate}

All 28 ST11 CRKP strains were isolated from blood culture samples collected for routine clinical examinations of hospitalized patients admitted to Ruijin Hospital in Shanghai, China, from 2018 to 2019. Any personally identifiable information was removed from this study. This study protocol, including the waiver of informed consent, was approved by the Ethics Committee of Ruijin Hospital, School of Medicine, Shanghai Jiao Tong University (RJ2019NO1-3). The research involved no more than minimal risk to subjects and no personal information was obtained. The research conformed to the principles of the Helsinki Declaration.

\section{Consent for publication}

Not applicable.

\section{Competing interests}

The authors declare that they have no competing interests.

\section{Author details}

'Department of Pulmonary and Critical Care Medicine, Ruijin Hospital, Shanghai Jiao Tong University School of Medicine, Shanghai 200025, China; Institute of Respiratory Diseases, Shanghai Jiao Tong University School of Medicine, Shanghai 200025, China; Shanghai Key Laboratory of Emergency Prevention, Diagnosis and Treatment of Respiratory Infectious Diseases, Shanghai 200025, China. ${ }^{2}$ State Key Laboratory of Microbial Metabolism, Joint International Laboratory on Metabolic \& Developmental Sciences, School of Life Sciences \& Biotechnology, Shanghai Jiao Tong University, Shanghai 200030, China. ${ }^{3}$ Department of Critical Care Medicine, Ruijin Hospital, Shanghai Jiao Tong University School of Medicine, Shanghai 200025, China. ${ }^{4}$ Department of Laboratory Medicine, Ruijin Hospital, Shanghai Jiao Tong
University School of Medicine, Shanghai 200025, China. ${ }^{5}$ Department of Clinical Microbiology, Ruijin Hospital, Shanghai Jiao Tong University School of Medicine, Shanghai 200025, China.

Received: 3 March 2021 Accepted: 9 July 2021

Published online: 22 July 2021

\section{References}

1. Navon-Venezia S, Kondratyeva K, Carattoli A. Klebsiella pneumoniae: a major worldwide source and shuttle for antibiotic resistance. FEMS Microbiol Rev. 2017:41(3):252-75. https://doi.org/10.1093/femsre/fux013.

2. Yang X, Dong N, Chan EW-C, Zhang R, Chen S. Carbapenem resistanceencoding and virulence-encoding conjugative plasmids in Klebsiella pneumoniae. Trends Microbiol. 2020.

3. Heiden SE, Hübner N-O, Bohnert JA, Heidecke C-D, Kramer A, Balau V, et al. A Klebsiella pneumoniae ST307 outbreak clone from Germany demonstrates features of extensive drug resistance, hypermucoviscosity, and enhanced iron acquisition. Genome Med. 2020;12(1):113. https://doi.org/10.1186/s13 073-020-00814-6.

4. Russo TA, Marr CM. Hypervirulent Klebsiella pneumoniae. Clin Microbiol Rev. 2019;32(3). https://doi.org/10.1128/CMR.00001-19.

5. Chen Y, Chang H, Lai Y, Pan C, Tsai S, Peng H. Klebsiella pneumoniae CG43 plasmid pLVPK, complete sequence. GenBank AY378100: NCBI Nucleotide; 2020. https://www.ncbi.nlm.nih.gov/nuccore/AY378100

6. Chen Y-T, Chang H-Y, Lai Y-C, Pan C-C, Tsai S-F, Peng H-L. Sequencing and analysis of the large virulence plasmid pLVPK of Klebsiella pneumoniae CG43. Gene. 2004;337:189-98.

7. Russo TA, Olson R, Fang C-T, Stoesser N, Miller M, MacDonald U, et al. Identification of biomarkers for differentiation of hypervirulent Klebsiella pneumoniae from classical K. pneumoniae. J Clin Microbiol. 2018;56(9). https://doi.org/10.1128/JCM.00776-18.

8. Lam MMC, Wyres KL, Wick RR, Judd LM, Fostervold A, Holt KE, et al. Convergence of virulence and MDR in a single plasmid vector in MDR Klebsiella pneumoniae ST15. J Antimicrob Chemother. 2019;74(5):1218-22. https://doi.org/10.1093/jac/dkz028.

9. Gu D, Dong N, Zheng Z, Lin D, Huang M, Wang L, et al. A fatal outbreak of ST11 carbapenem-resistant hypervirulent Klebsiella pneumoniae in a Chinese hospital: a molecular epidemiological study. Lancet Infect Dis. 2018;18(1):3746. https://doi.org/10.1016/S1473-3099(17)30489-9.

10. Xie Y, Tian L, Li G, Qu H, Sun J, Liang W, et al. Emergence of the thirdgeneration cephalosporin-resistant hypervirulent Klebsiella pneumoniae due to the acquisition of a self-transferable $b / a_{\mathrm{DHA}-1}$-carrying plasmid by an ST23 strain. Virulence. 2018:838-44.

11. Zhang R, Lin D, Chan EW-C, Gu D, Chen G-X, Chen S. Emergence of Carbapenem-resistant serotype K1 hypervirulent Klebsiella pneumoniae strains in China. Antimicrob Agents Chemother. 2016;60(1):709-11. https:// doi.org/10.1128/AAC.02173-15.

12. Turton J, Davies F, Turton J, Perry C, Payne Z, Pike R. Hybrid resistance and virulence plasmids in "high-risk" clones of Klebsiella pneumoniae, including

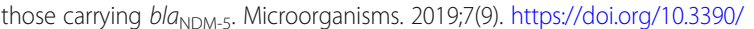
microorganisms7090326.

13. Dong N, Lin D, Zhang R, Chan EW-C, Chen S. Carriage of bla $a_{\mathrm{KPC}-2}$ by a virulence plasmid in hypervirulent Klebsiella pneumoniae. J Antimicrob Chemother. 2018;73:3317-21.

14. Huang Y-H, Chou S-H, Liang S-W, Ni C-E, Lin Y-T, Huang Y-W, et al. Emergence of an XDR and carbapenemase-producing hypervirulent Klebsiella pneumoniae strain in Taiwan. J Antimicrob Chemother. 2018;73(8): 2039-46. https://doi.org/10.1093/jac/dky164.

15. Yang X, Wai-Chi Chan E, Zhang R, Chen S. A conjugative plasmid that augments virulence in Klebsiella pneumoniae. Nat Microbiol. 2019;4(12): 2039-43. https://doi.org/10.1038/s41564-019-0566-7.

16. Xie M, Chen $K$, Ye L, Yang X, Xu Q, Yang C, et al. Conjugation of virulence plasmid in clinical Klebsiella pneumoniae strains through formation of a fusion plasmid. Adv Biosyst. 2020;4:e1900239.

17. Li R, Cheng J, Dong H, Li L, Liu W, Zhang C, et al. Emergence of a nove conjugative hybrid virulence multidrug-resistant plasmid in extensively drug-resistant Klebsiella pneumoniae ST15. Int J Antimicrob Agents. 2020;55: 105952.

18. Qu H, Ou H. Klebsiella pneumoniae subsp. pneumoniae strain RJF293 plasmid pRJF293, complete sequence. BioProject PRJNA307277. NCBI 
Nucleotide. 2016; https://www.ncbi.nlm.nih.gov/nuccore/?term=PRJNA3072 77.

19. Wang X, Xie Y, Li G, Liu J, Li X, Tian L, et al. Whole-genome-sequencing characterization of bloodstream infection-causing hypervirulent Klebsiella pneumoniae of capsular serotype K2 and ST374. Virulence. 2018;9(1):510-21. https://doi.org/10.1080/21505594.2017.1421894.

20. Bi D, Jiang X, Sheng Z-K, Ngmenterebo D, Tai C, Wang M, et al. Mapping the resistance-associated mobilome of a carbapenem-resistant Klebsiella pneumoniae strain reveals insights into factors shaping these regions and facilitates generation of a "resistance-disarmed" model organism. J Antimicrob Chemother. 2015;70(10):2770-4. https://doi.org/10.1093/jac/dkv2 04.

21. Koren S, Walenz BP, Berlin K, Miller JR, Bergman NH, Phillippy AM. Canu: scalable and accurate long-read assembly via adaptive $k$-mer weighting and repeat separation. Genome Res. 2017;27(5):722-36. https://doi.org/10.1101/ gr.215087.116.

22. Qu J, Ou H. Klebsiella pneumoniae subsp. pneumoniae strain:RJBSI76 genome sequencing. BioProject PRJNA681750. NCBI Nucleotide. 2021; https://www.ncbi.nlm.nih.gov/nuccore/?term=PRJNA681750.

23. Qu J, Ou H. Klebsiella pneumoniae subsp. pneumoniae strain:RJBSI76-pV genome sequencing. BioProject PRJNA682095. NCBI Nucleotide. 2021; https://www.ncbi.nlm.nih.gov/nuccore/?term=PRJNA682095.

24. Qu J, Ou H. Escherichia coli strain:J53-p1-pV-hybrid-1 genome sequencing. BioProject PRJNA692573. NCBI Nucleotide. 2021; https://www.ncbi.nlm.nih. gov/nuccore/?term=PRJNA692573.

25. Qu J, Ou H. Escherichia coli strain:XL10-pF-pV-hybrid-1 genome sequencing BioProject PRJNA692574. NCBI Nucleotide. 2021; https://www.ncbi.nlm.nih. gov/nuccore/1968731931.

26. Seemann T. Prokka: rapid prokaryotic genome annotation. Bioinformatics. 2014;30(14):2068-9. https://doi.org/10.1093/bioinformatics/btu153.

27. Carattoli A, Zankari E, García-Fernández A, Voldby Larsen M, Lund O, Villa L, et al. In silico detection and typing of plasmids using PlasmidFinder and plasmid multilocus sequence typing. Antimicrob Agents Chemother. 2014; 58(7):3895-903. https://doi.org/10.1128/AAC.02412-14.

28. Lam MMC, Wick RR, Wyres KL, Holt KE. Genomic surveillance framework and global population structure for Klebsiella pneumoniae. bioRxiv. 2020; 2020.12. 14.422303 .

29. Li J, Tai C, Deng Z, Zhong W, He Y, Ou H-Y. VRprofile: gene-clusterdetection-based profiling of virulence and antibiotic resistance traits encoded within genome sequences of pathogenic bacteria. Brief Bioinform. 2018;19:566-74.

30. Li X, Xie Y, Liu M, Tai C, Sun J, Deng Z, et al. oriTfinder: a web-based tool for the identification of origin of transfers in DNA sequences of bacterial mobile genetic elements. Nucleic Acids Res. 2018;46:W229-34.

31. Alikhan N-F, Petty NK, Ben Zakour NL, Beatson SA. BLAST Ring Image Generator (BRIG): simple prokaryote genome comparisons. BMC Genomics. 2011;12(1):402. https://doi.org/10.1186/1471-2164-12-402.

32. Sullivan MJ, Petty NK, Beatson SA. Easyfig: a genome comparison visualizer. Bioinformatics. 2011;27(7):1009-10. https://doi.org/10.1093/bioinformatics/ btr039.

33. Bailey TL, Boden M, Buske FA, Frith M, Grant CE, Clementi L, et al. MEME SUITE: tools for motif discovery and searching. Nucleic Acids Res. 2009; 37(Web Server):W202-8. https://doi.org/10.1093/nar/gkp335.

34. Palacios M, Broberg CA, Walker KA, Miller VL. A serendipitous mutation reveals the severe virulence defect of a Klebsiella pneumoniae fepB mutant. mSphere. 2017;2.

35. Li L, Stoeckert CJJ, Roos DS. OrthoMCL: identification of ortholog groups for eukaryotic genomes. Genome Res. 2003;13(9):2178-89. https://doi.org/10.11 01/gr.1224503.

36. Letunic I, Bork P. Interactive Tree Of Life (iTOL) v4: recent updates and new developments. Nucleic Acids Res. 2019;47(W1):W256-9. https://doi.org/10.1 093/nar/gkz239

37. Wyres KL, Wick RR, Judd LM, Froumine R, Tokolyi A, Gorrie CL, et al. Distinct evolutionary dynamics of horizontal gene transfer in drug resistant and virulent clones of Klebsiella pneumoniae. PLoS Genet. 2019;15(4):e1008114. https://doi.org/10.1371/journal.pgen.1008114.

38. Walker KA, Miner TA, Palacios M, Trzilova D, Frederick DR, Broberg CA, et al. A Klebsiella pneumoniae regulatory mutant has reduced capsule expression but retains hypermucoviscosity. MBio. 2019;10(2). https://doi.org/10.1128/ mBio.00089-19.
39. Walker KA, Treat LP, Sepúlveda VE, Miller VL. The small protein RmpD drives hypermucoviscosity in Klebsiella pneumoniae. MBio. 2020;11(5). https://doi. org/10.1128/mBio.01750-20.

40. Smillie C, Garcillán-Barcia MP, Francia MV, Rocha EPC, de la Cruz F. Mobility of plasmids. Microbiol Mol Biol Rev. 2010;74(3):434-52. https://doi.org/10.112 8/MMBR.00020-10.

41. Lawley TD, Klimke WA, Gubbins MJ, Frost LS. F factor conjugation is a true type IV secretion system. FEMS Microbiol Lett. 2003;224(1):1-15. https:/doi. org/10.1016/50378-1097(03)00430-0.

42. Furuya N, Nisioka T, Komano T. Nucleotide sequence and functions of the oriT operon in Incl1 plasmid R64. J Bacteriol. 1991;173(7):2231-7. https://doi. org/10.1128/jb.173.7.2231-2237.1991.

43. O'Connor MB, Kilbane JJ, Malamy MH. Site-specific and illegitimate recombination in the oriv1 region of the $\mathrm{F}$ factor. DNA sequences involved in recombination and resolution. J Mol Biol. 1986;189:85-102.

44. Kopotsa K, Osei Sekyere J, Mbelle NM. Plasmid evolution in carbapenemaseproducing Enterobacteriaceae: a review. Ann N Y Acad Sci. 2019;1457:61-91.

45. Bi D, Zheng J, Li J-J, Sheng Z-K, Zhu X, Ou H-Y, et al. In silico typing and comparative genomic analysis of IncFIl $K_{K}$ plasmids and insights into the evolution of replicons, plasmid backbones, and resistance determinant profiles. Antimicrob Agents Chemother. 2018;62(10). https://doi.org/10.112 8/AAC.00764-18.

46. Shu L, Dong N, Lu J, Zheng Z, Hu J, Zeng W, et al. Emergence of OXA-232 carbapenemase-producing Klebsiella pneumoniae that carries a pLVPK-Like virulence plasmid among elderly patients in China. Antimicrob Agents Chemother. 2019;63(3). https://doi.org/10.1128/AAC.02246-18.

47. Wong MH-Y, Chan EW-C, Chen S. IS26-mediated formation of a virulence and resistance plasmid in Salmonella enteritidis. J Antimicrob Chemother. 2017;72(10):2750-4. https://doi.org/10.1093/jac/dkx238.

48. Bouet J-Y, Bouvier M, Lane D. Concerted action of plasmid maintenance functions: partition complexes create a requirement for dimer resolution. Mol Microbiol. 2006;62(5):1447-59. https://doi.org/10.1111/j.1365-2958.2006. 05454.x.

49. Liu G, Bogaj K, Bortolaia V, Olsen JE, Thomsen LE. Antibiotic-induced, increased conjugative transfer is common to diverse naturally occurring ESBL plasmids in Escherichia coli. Front Microbiol. 2019;10:2119. https://doi. org/10.3389/fmicb.2019.02119.

50. Kim S, Yun Z, Ha U-H, Lee S, Park H, Kwon EE, et al. Transfer of antibiotic resistance plasmids in pure and activated sludge cultures in the presence of environmentally representative micro-contaminant concentrations. Sci Total Environ. 2014;468-469:813-20.

51. Anthony KG, Sherburne C, Sherburne R, Frost LS. The role of the pilus in recipient cell recognition during bacterial conjugation mediated by F-like plasmids. Mol Microbiol. 1994;13(6):939-53. https://doi.org/10.1111/j.1365-2 958.1994.tb00486.x.

52. Pérez-Mendoza $D$, de la Cruz F. Escherichia coli genes affecting recipient ability in plasmid conjugation: are there any? BMC Genomics. 2009;10(1):71. https://doi.org/10.1186/1471-2164-10-71.

53. Fang C-T, Chuang Y-P, Shun C-T, Chang S-C, Wang J-T. A novel virulence gene in Klebsiella pneumoniae strains causing primary liver abscess and septic metastatic complications. J Exp Med. 2004;199(5):697-705. https://doi. org/10.1084/jem.20030857.

54. The NCBI. Nucleotide/Protein database. National Center for. Biotechnol Inform (NCBI). https://www.ncbi.nlm.nih.gov/nucleotide/. Accessed 3 Mar 2021

\section{Publisher's Note}

Springer Nature remains neutral with regard to jurisdictional claims in published maps and institutional affiliations. 